\title{
Geografía de los desahucios por ejecución hipotecaria en las ciudades españolas: evidencias a partir de las viviendas propiedad de la SAREB ${ }^{1}$
}

\author{
Aaron Gutiérrez² y Antoni Domènech ${ }^{3}$
}

\begin{abstract}
RESUMEN
El estallido de la burbuja inmobiliaria y el inicio de la crisis financiero-inmobiliaria se han traducido, en España, en la mayor proliferación de las ejecuciones hipotecarias y desahucios de Europa occidental. Ello pone en evidencia las lógicas depredadoras del modelo inmobiliario español, el cual ha alimentado un gigantesco proceso de desposesión de vivienda. El artículo aporta evidencias del desigual desarrollo de la crisis hipotecaria en las ciudades españolas a partir de la explotación de datos del stock de vivienda en manos de la SAREB proveniente de ejecuciones hipotecarias. El análisis territorial de la distribución de las viviendas propiedad de la SAREB en tres ciudades (Alicante, Murcia y Zaragoza) permite identificar los barrios con una especial afectación de procesos de desposesión de vivienda. Los resultados demuestran como las ejecuciones hipotecarias están tendiendo a concentrarse en los barrios más vulnerables, profundizando su situación de precariedad. Ello evidencia como la crisis financiero-inmobiliaria está contribuyendo a profundizar las desigualdades urbanas.
\end{abstract}

Palabras clave: SAREB, ejecuciones hipotecarias, desahucios, desigualdad, España.

\begin{abstract}
The bursting of the housing bubble and the onset of the financial and real estate crisis have resulted, in Spain, in the biggest proliferation of foreclosures and evictions than any Western European countries. That situation reveals the predatory logic of the Spanish real estate model, which has fuelled a huge housing dispossession process. The article provides evidences of the uneven development of the mortgage crisis in the Spanish cities. The study is based on data exploitation of housing stock in the hands of the SAREB acquired through foreclosures. The spatial analysis of the distribution of housing units owned by SAREB in three cities (Alicante, Murcia and Zaragoza) has allowed identifying neighbourhoods with a special concentration of housing dispossession processes. The results show how foreclosures are tending to focus on the most vulnerable neighbourhoods, which translates to a deepening of their precarious situation. These findings show how the financial and real estate crisis are contributing to deepening urban inequality.
\end{abstract}

Keywords: SAREB, foreclosures, evictions, inequality, Spain. 
La proliferación de los desahucios a partir del estallido de la burbuja inmobiliaria y la crisis económica y financiera global es una de las manifestaciones más evidentes de las implicaciones del modelo inmobiliario y financiero español (Burriel, 2014; Colau y Alemany, 2012; López y Rodríguez, 2010; Valiño et al., 2013; Vinuesa, 2013). Modelo que parte de la concepción de la vivienda como un bien de inversión y un instrumento de generación y apropiación de rentas (Gaja, 2013; Vives-Miró y Rullan, 2014). Los datos demuestran que en España la magnitud del crecimiento de los desahucios desde 2009 es superior al resto de Estados europeos (Cano et al., 2013). Ello ha derivado en un agudo proceso de desposesión de vivienda y empobrecimiento de la sociedad española. En este contexto, los bancos han jugado un papel protagonista, a través de una legislación hipotecaria que lo ha propiciado, ya que una parte substancial de estos desahucios se derivan de ejecuciones hipotecarias causadas por el impago de los créditos hipotecarios contraídos ${ }^{4}$. Según datos del Consejo General del Poder Judicial, entre 2008 y 2014 en España se han realizado cerca de 600.000 ejecuciones hipotecarias. Estas ejecuciones se han traducido en cerca de 380.000 lanzamientos durante el mismo período. Sin embargo, no existen datos oficiales, y que abarquen todo el período, del número total de desahucios de vivienda habitual que estas ejecuciones han causado; aunque, evidentemente, las cifras denotan que se trata de una cifra elevadísima.

Esta extensión de los desahucios por impago del crédito hipotecario ha sido acompañada, y alimentada, por los llamados procesos de "reestructuración y saneamiento" del sector financiero español. Esto es, el rescate de la banca con dinero público y la bancarización de las cajas de ahorro regionales (reestructuración) más el trasvase de activos provenientes del sector inmobiliario (suelo, promociones de vivienda vacía y viviendas adquiridas a través de ejecución hipotecaria) a la SAREB (saneamiento). Lo más significativo de este proceso es que los bancos rescatados con dinero público han sido, precisamente, aquellos que más han desahuciado. Los datos disponibles en Cataluña permiten ilustrar que cerca de un $65 \%$ de los desahucios derivados de una ejecución hipotecaria han sido propiciados por las entidades financieras rescatadas con dinero público (Gutiérrez y Delclòs, 2017). La creación de la SAREB en 2012 (Sociedad de Gestión de Activos Procedentes de la Reestructuración Bancaria) ha sido una pieza fundamental en este proceso, ya que ha adquirido un aporte importante de la vivienda de estos bancos rescatados. Esta sociedad pretende "ayudar al saneamiento del sector financiero, y en concreto de las entidades que arrastraban problemas debido a su excesiva exposición al sector inmobiliario, con el objetivo de reducir los riesgos de las mismas y liquidar de forma ordenada los activos problemáticos" ${ }^{\prime 5}$. Es decir, adquiere las viviendas de los bancos para después venderlas a particulares, empresas o sociedades de inversión (como fondos buitre ${ }^{6}$ ).

Dada la relevancia social, política y económica de este fenómeno, existe una creciente literatura académica que analiza la problemática de los desahucios desde diferentes perspectivas. A modo de síntesis, podemos destacar los estudios que, desde la óptica económica y financiera, detallan y critican el modelo que los alimenta (Coq-Huelva, 2013; Hoekstra \& Vakili-zad, 2011);

\footnotetext{
En el inicio de la crisis financiera (entre 2008 y 2010) los desahucios vinculados a ejecuciones hipotecarias representaban cerca del $90 \%$ del total, pero la tendencia en los años siguientes ha sido el aumento de los derivados del impago del alquiler. Así, éstos ya alcanzaron el $57 \%$ del total en 2013 (datos del Consejo General del Poder Judicial).

https://www.sareb.es/es-es/SobreSareb/Paginas/que-es-sareb.aspx

Los fondos buitre, también conocidos como fondos de inversión libre o fondos especializados en activos en dificultades, tienen como principal objetivo comprar activos a Estados, empresas y entidades financieras en riesgo de quiebra a un precio más reducido al de su valor nominal para litigarlos o venderlos en un periodo de tiempo razonable y conseguir altas rentabilidades.
} 
también aquellos que enfatizan su contribución a la precarización social (Colau y Alemany, 2012; Valiño et al., 2013); otros analizan los movimientos sociales de lucha y resistencia (Álvarez et al., 2015; Barbero, 2015; Romanos, 2014) o su impacto sobre la calidad de vida y la salud de las familias (Gili et al., 2012; Valiño et al., 2015). Sin embargo, existe un déficit de estudios que analicen la proliferación de los desahucios por ejecución hipotecaria a partir de una perspectiva geográfica. Este déficit es relevante, porqué los desahucios, como cualquier fenómeno socioeconómico, también deben ser analizados a partir de sus pautas territoriales. Esta casuística se explica, básicamente, por la ausencia de fuentes válidas para el estudio de los desahucios a escala urbana. Las fuentes oficiales proveen información agregada por provincias (Instituto Nacional de Estadística) o Partidos Judiciales (Consejo General del Poder Judicial). Existen estudios publicados que explotan estas fuentes (Méndez y Plaza 2016; Obeso, 2014), permitiendo una lectura geográfica para el conjunto del Estado a partir de los datos de los 431 partidos judiciales, pero no posibilitan un análisis de la desigual distribución de los desahucios en las ciudades españolas, ya que su nivel de agregación no es a escala municipal y/o inframunicipal. Vives-Miró et al. (2015), Gutiérrez \& Delclòs (2016) y Gutiérrez \& Domènech (2017) han explotado fuentes diferentes que facultan esta aproximación a escala urbana del fenómeno aplicado a sendos casos de estudio. El trabajo de Vives-Miró et al. (2015) se desarrolló en base a la recopilación de datos del Servició Común de Notificaciones y Embargos de Palma que permitió representar los desahucios en el partido judicial de Palma de Mallorca. Por su parte, Gutiérrez \& Delclòs (2016) y Gutiérrez \& Domènech (2017), a partir de un vaciado de los portales inmobiliarios de las filiales inmobiliarias de los bancos, georreferenciaron las viviendas en manos de la banca adquiridas a través de ejecución hipotecaria. El presente trabajo permite combinar ambas aproximaciones: una visión general para el conjunto de España, combinada con una profundización en estudios de caso. A partir de una explotación de fuentes secundarias, se identifica la desigual distribución en el conjunto de España de las viviendas en manos de la SAREB adquiridas vía ejecución hipotecaria y desahucio. A su vez, se profundiza en diferentes estudios de caso para analizar las lógicas de su distribución a escala urbana.

El trabajo parte de dos objetivos, uno instrumental o metodológico, y otro de investigación. Por lo que al objetivo metodológico se refiere, el artículo pretende aportar una solución innovadora al déficit crónico de datos relativos a las ejecuciones hipotecarias y los desahucios a una escala territorial que proporciona mayor detalle que los partidos judiciales. Por ello se ha utilizado una fuente alternativa (el vaciado sistematizado de portales inmobiliarios) que permite trabajar hasta el detalle de la dirección postal de cada vivienda georreferenciada; por lo que las posibilidades de análisis espacial a diferentes escalas son múltiples. En este sentido, el trabajo se plantea, también, como una validación de la fuente de datos planteada.

El objetivo de investigación es analizar y caracterizar la distribución espacial de las viviendas en manos de la SAREB a diferentes escalas territoriales (el conjunto del territorio español y diferentes áreas urbanas utilizadas como casos de estudio). Ello debe permitir elaborar una detallada cartografía de la desposesión de vivienda en España y de la acumulación en manos de un nuevo gran propietario: la SAREB. En relación con el objetivo de investigación el artículo parte de las siguientes hipótesis:

1. La distribución espacial de las viviendas en manos de la SAREB no es aleatoria, sino que es un reflejo de aquellas áreas urbanas que han sido más afectadas por la crisis hipotecaria. 
2. Las áreas urbanas donde se concentran los desahucios son, precisamente, los barrios más vulnerables. Aquellos que concentran las clases populares, los menores niveles de renda y las viviendas de menor calidad. De ello se deriva es que la crisis hipotecaria española está contribuyendo, a profundizar la desigualdad urbana, ya que afecta especialmente a las familias y barrios con menos recursos.

A continuación de esta introducción se presenta el contexto de referencia en el que se enmarca la investigación: el modelo financiero e inmobiliario español y su culminación en la crisis hipotecaria española. Posteriormente, se presenta como nace en este contexto la SAREB y las lógicas financiero-inmobiliarias que alimenta. A continuación se presenta y justifica los datos utilizados en la investigación y los métodos utilizados para explotarlos. Finalmente, se presentan y discuten los resultados obtenidos y las conclusiones que de éstos se derivan.

\section{Contexto: Crisis hipotecaria española y su contribución a la desigualdad urbana}

El contexto global de financiarización de la economía, vivida a partir de los años setenta del siglo XX (Lapavitsas, 2011), se tradujo, especialmente a partir de los noventa, en una financiarización del entorno construido (Lapavistsas, 2009; Christophers, 2015) y en la galopante especulación inmobiliaria apoyada en la conceptualización de la vivienda como un motor de creación y apropiación de rentas (Vives-Miró y Rullan, 2014). En otros términos, es aquello que varios autores han conceptualizado como financiarización de la vivienda (Aalbers, 2008; Rolnik, 2013). De hecho, el "Modelo Español" (López \& Rodríguez, 2011) supone uno de los ejemplos más evidentes de la expansión del circuito secundario de acumulación de capital centrado en la producción del espacio urbano y, concretamente, en la vivienda (Gotham, 2009).

La actual crisis hipotecaria se enmarca en un modelo inmobiliario y financiero español con raíces políticas y económicas arraigadas hace ya varias décadas (Fernández Durán, 2006; Naredo, 2010; Romero et al., 2012). Es decir, los desahucios no son un hecho aislado, sino una pieza más de unas políticas económicas que parten del fomento de la sociedad de propietarios promovida por el régimen franquista. Los gobiernos democráticos no reorientaron las políticas públicas hacia el fomento del alquiler (López y Rodríguez, 2010), lo que ha comportado que, actualmente, España sea el estado de Europa Occidental con mayor peso de la vivienda en propiedad (más de un $80 \%$ según datos de Eurostat); mientras que hace cinco décadas la vivienda en propiedad no alcanzaba el $50 \%$.

No obstante, aunque la lógica que subyace en el modelo no sea nueva, el boom inmobiliario y la burbuja de precios vividos durante la década 1997-2007 lo exacerbaron (Fernández y Cruz, 2011; Gutiérrez y Delclòs, 2015). El capital financiero global, alimentado por la creciente desregulación, y a la búsqueda de inversiones en productos que aportaran altas rentabilidades a corto plazo, encontró en el sector inmobiliario español un producto muy apetecible. En un contexto global de financiarización de la economía, el capital encontró en la producción inmobiliaria una vía de especulación, generación y apropiación de rentas (Aalbers, 2008), engrosando el circuito secundario de acumulación (Gotham, 2009). 
En un contexto donde la hipoteca se había consolidado como fórmula principal de acceso a la vivienda, el endeudamiento de las familias creció exponencialmente. El aumento de los años de amortización de los créditos hipotecarios, sumado a la reducción de tipos de interés, facilitó que pese al continuo incremento de los precios y el estancamiento de las rentas del trabajo, el número de familias hipotecadas creciera año a año. Así, según datos del Banco de España analizados por el Observatorio Metropolitano de Madrid, durante el período 1994-2007 se multiplicó por doce el volumen total de endeudamiento hipotecario en España; además, el volumen total de la deuda privada (familias y empresas) se multiplicó por tres (López y Rodríguez, 2010).

A partir del estallido de la burbuja inmobiliaria, la caída de precios y el inicio de la crisis financiera y económica, se ha iniciado un proceso estrechamente ligado con el anterior: la proliferación de los desahucios. Ambos ciclos forman parte de la misma lógica que alimenta la financiarización del entorno construido y la apropiación de rentas a través de la desposesión. El rápido ascenso del paro, sumado a la sobreexposición al crédito de las familias ha derivado en la imposibilidad del pago de las deudas contraídas y, con ello, la explosión de la crisis de los desahucios (Gutiérrez y Delclòs, 2017). Esta situación no ha afectado por igual a toda la sociedad, sino que ha tendido a golpear con mayor intensidad a las clases populares (Colau y Alemany, 2012; Valiño et al., 2015). Ha alimentado la precarización y ha incrementado las desigualdades, cebándose en los más vulnerables.

\section{La SAREB como culminación del despropósito financiero- inmobiliario español}

La SAREB, conocida popularmente como el "banco malo", pese a no ser un banco propiamente, es una sociedad creada en noviembre de 2012 fruto de los acuerdos del Memorandum of Understanding (MoU) firmado entre el estado español y la Comisión Europea (RDL 24/2012 y Real Decreto 1559/2012) en el marco del rescate a la banca española. El Fondo de Reestructuración Ordenada Bancaria (FROB) 7 es principal accionista de la SAREB. Dispone de un $45 \%$ del capital y aportó 2.192 millones de euros de dinero público a la sociedad. El 55\% del capital restante es privado y se reparte entre 27 sociedades, esencialmente entidades financieras.

EI MoU definió los mecanismos para la constitución de la SAREB y los objetivos que debían guiar el proceso. Éstos son, en primer lugar, disponer de una entidad, un "banco malo", con capacidad de recibir ayuda financiera y, en segundo lugar, transferir los activos inmobiliarios de las entidades que atravesaban dificultades a esta sociedad. Todo ello permite reducir sus riesgos y liquidar de forma ordenada los activos 'tóxicos' acumulados a consecuencia de sus malas decisiones crediticias. Estos activos incluyen los diferentes productos vinculados con el sector inmobiliario, básicamente, deuda a promotores de difícil cobro, suelo (en algunos casos ya urbanizado), promociones de vivienda vacía (en algunos casos sin acabar de construir) y viviendas adquiridas a través de ejecución hipotecaria. Nuestro trabajo se centra en el tercero de estos activos: las

El FROB es un fondo creado por el Real Decreto-Ley 9/2009. En el contexto de la crisis financiera, el fondo nace con dos objetivos: gestionar los procesos de reestructuración de las entidades financieras y reforzarlas con aportaciones de capital durante los procesos de integración. Inicialmente, es dotado con 9.000 millones de euros, el 75\% de los cuáles a cargo de los presupuestos generales del Estado y el 25\% restante a cargo del Fondo de Adquisición de Activos Financieros. 
viviendas que se han adjudicado los bancos desahuciando las familias residentes y que, posteriormente, han pasado a manos de la SAREB. Ésta ahora pretende venderlas, completando así el círculo de acumulación por desposesión (Harvey, 2003); es decir, expolio generalizado.

En 2012 se realizó la transferencia de los activos "tóxicos" de las entidades financieras nacionalizadas (Bankia, Catalunya Caixa, Novacaixa Galicia y Banco de Valencia) con un valor aproximado de 44.000 millones de euros, pagados a través de garantías que el Estado transfirió a la SAREB. Posteriormente, en 2013, se transfirió los activos de las entidades financieras en proceso de reestructuración y absorción por otras entidades (BMN, Ceiss, Liberbank y Caja3) por 21.000 millones de euros más, también a través de garantías del Estado. Los bancos cambiaron estas garantías por liquidez del Banco Central Europeo, por lo que al tratarse de entidades que previamente habían sido rescatadas, han sido entidades doblemente intervenidas con dinero público.

El compromiso de la SAREB es vender todos estos activos en un plazo de quince años desde su constitución. Los paquetes inmobiliarios son vendidos, principalmente, fondos de inversión (los llamados fondos buitre), aunque también se promueve la venta de inmuebles a particulares y sociedades. Las garantías firmadas por la sociedad y las entidades financieras implican que si en estos quince años la SAREB no alcanza este objetivo el Estado deberá asumir las pérdidas económicas que de ello se deriven. Todo ello resulta en la acumulación de ayudas públicas en una estructura concebida para que inversores privados (básicamente fondos buitre) rentabilicen la reventa de, entre otros productos, viviendas adquiridas a través del desalojo de las familias residentes. En el caso que esta reventa no resulte rentable, el Estado responderá con más dinero público.

\section{Datos y métodos}

El objetivo instrumental del presente trabajo es identificar y explotar una fuente alternativa que permita el estudio de los desahucios por ejecución hipotecaria a escala urbana, paliando el déficit de información derivado de la agregación estadística a nivel de partidos judiciales. Para ello se ha utilizado los datos de viviendas de segunda mano propiedad de la SAREB. Como se ha comentado anteriormente, la SAREB acumula diferentes tipos de activos vinculados con el sector inmobiliario; esencialmente, suelo, edificios de obra nueva vacía sin vender y viviendas adquiridas a través de ejecuciones hipotecarias. Los activos de interés para los objetivos de esta investigación son las viviendas adquiridas a través de ejecución hipotecaria que han desembocado en un desahucio. Estas viviendas son comercializadas a través de los portales web de las filiales inmobiliarias de los bancos que han firmado acuerdos de comercialización con la SAREB (Altamira, Haya, Servihabitat y Solvia ${ }^{8}$ ). Durante los últimos años estos portales web se han popularizado como vía de publicidad para la comercialización (en alquiler o venda) de las viviendas en manos de los bancos en España. Ahora la SAREB se sirve de estas plataformas para publicitar las viviendas que pretende vender, incorporando su logo en aquellas viviendas de las cuales es titular. Para el desarrollo del presente trabajo se han seleccionado las viviendas clasificadas como segunda mano. Éstas se corresponden con las que han sido adquiridas por los bancos a través procesos de 
ejecución hipotecaria que derivan en un desahucio (a diferencia de las viviendas de obra nueva sin primera ocupación).

En estos portales, se ofrece información, en forma de anuncio, de cada vivienda en venda donde se incluye la dirección postal y un mapa de ubicación. A partir de estos datos se ha georreferenciado cada vivienda identificada, hasta un total de 33.205 viviendas. Al ser georreferenciadas individualmente, se posibilitan los análisis espaciales a cualquier escala. La base confeccionada permite identificar detalladamente qué activos posee la SAREB y cuál es su distribución territorial. Pero puede ser utilizada también como una muestra altamente representativa de la distribución territorial de los desahucios producidos por ejecución hipotecaria en España. Como se ha comentado anteriormente, la SAREB ha adquirido activos inmobiliarios de múltiples bancos, por lo que finalmente el stock acumulado representa una buena radiografía del conjunto. Por ello, el presente trabajo también interpreta los datos generados como una representación de los desahucios por ejecución hipotecaria en España. Es decir, se dispone de una muestra de 33.205 unidades que permiten caracterizar las pautas territoriales del conjunto.

El vaciado sistematizado de los portales inmobiliarios se desarrolló durante cinco meses entre noviembre de 2015 y marzo de 2016. Por lo que los datos presentados corresponden al stock de vivienda de segunda mano acumulado por la SAREB en marzo de 2016.

Una vez georreferenciadas las viviendas de segunda mano propiedad de la SAREB (VSMS), se han realizado diferentes procesos para permitir su análisis espacial. En primer lugar, se han agregado los valores (número de VSMS) por diferentes unidades territoriales a través del SIG. El trabajo ha utilizado las agregaciones del total de VSMS por municipios y Comunidades Autónomas. Estos datos, a su vez, se han ponderado en relación al volumen de habitantes y número de viviendas de cada unidad territorial de análisis. De forma, que se expresa el número de VSMS por cada 1.000 habitantes y cada 1.000 viviendas, lo que permite un análisis comparado entre territorios.

Más allá del análisis de la distribución territorial de las VSMS en el conjunto del Estado, el objetivo del trabajo es analizar sus pautas espaciales a escala urbana. Para ello se han seleccionado tres ciudades como estudios de caso: Alicante (Comunidad Valenciana), Murcia (Región de Murcia) y Zaragoza (Aragón). Su elección se basa en diferentes factores. En primer lugar, se debe a que las tres ciudades concentran un número elevado de VSMS, situándose entre los 25 municipios con mayor volumen. En segundo lugar, son ciudades de un volumen demográfico importante, por lo que disponen de datos sociodemográficos desagregados por secciones censales. La calidad del censo de población de 2011 (elaborado por muestreo) no permite disponer de datos sociodemográficos a escala inframunicipial en ciudades pequeñas. Por lo que, si se pretende trabajar con datos por secciones censales, como es el caso, se requiere de ciudades relativamente grandes. En tercer lugar, se ha utilizado un criterio territorial. Se han seleccionado ciudades pertenecientes a diferentes Comunidades Autónomas, que permitan presentar contextos donde los bancos $y$, especialmente, las cajas de ahorros regionales que protagonizaron las ejecuciones hipotecarias eran diferentes, por lo que se introducen elementos que permiten diversificar la muestra. El Cuadro No 1 resume los datos de VSMS en cada una de las ciudades objeto de estudio. 
Cuadro No 1

Viviendas propiedad de la SAREB - Alicante, Murcia y Zaragoza

\begin{tabular}{|l|r|r|r|}
\hline & \multicolumn{1}{|c|}{ Alicante } & \multicolumn{1}{|c|}{ Murcia } & \multicolumn{2}{c|}{ Zaragoza } \\
\hline No VSMS & 405 & 454 & 295 \\
\hline Habitantes 2011 & 329.325 & 437.667 & 678.115 \\
\hline VSMS por 1.000 habitantes & 1,2 & 1,0 & 0,4 \\
\hline Viviendas 2011 & 106.880 & 112.255 & 244.365 \\
\hline VSMS por 1.000 viviendas & 3,8 & 4,0 & 1,2 \\
\hline
\end{tabular}

Fuente: Elaboración propia a partir de datos de los portales inmobiliarios de los bancos y del Censo de Población y Vivienda de 2011 (Instituto Nacional de Estadística).

A través de estos casos de estudio se ha desarrollado el análisis territorial que permite validar o rechazar las dos hipótesis de investigación planteadas. Para validar o rechazar la primera de las hipótesis del trabajo (sobre la concentración de los desahucios en determinadas áreas urbanas), se ha realizado un análisis de la clusterización de las VSMS. Para ello se ha aplicado el estadístico espacial Gi* de Getis-Ord mediante el programario de ESRI ArcGIS 10.3 @. Se trata de un cálculo que se desarrolla teniendo en cuenta los valores de cada entidad considerando también los valores de sus entidades vecinas. El análisis de clusterización se ha desarrollado usando las manzanas como unidad básica de análisis y agregando en éstas el total de VSMS contenidas. Se ha definido una distancia fija de 300 metros como unidad de cálculo para identificar relaciones espaciales de concentración o dispersión de valores por zonas. La herramienta devuelve una puntuación z para cada entidad del dataset (por cada manzana), la cual puede ser significativamente positiva o negativa con un nivel de confianza mínimo del 90\%. La estadística Gi* de Getis-Ord se define como:

Paso 1:

$$
G_{i}^{*}=\frac{\sum_{j=1}^{n} x_{i, j} x_{j}-\bar{X} \sum_{j=1}^{n} w_{i, j}}{\sqrt{\frac{\left[n \sum_{j=1}^{n} w_{i, j}^{2}-\left(\sum_{j=1}^{n} w_{i, j}\right)_{i}^{2}\right]}{n-1}}}
$$

Donde $x_{j}$ es el valor del atributo para la entidad $j, w_{i j}$ es el peso espacial entre la entidad ${ }_{i} y_{j}, n$ es igual al número total de entidades $y$ :

Paso 2:

Paso 3:

$$
\bar{X}=\frac{\sum_{j=1}^{n} x_{j}}{n}
$$

$$
S=\sqrt{\sum_{j=}^{n} x_{j}^{2}-(\bar{X})^{2}}
$$

Los barrios con una mayor concentración de VSMS son identificados a partir de la concentración de manzanas con unas puntuaciones z positivas estadísticamente significativas; mientras más grande es la puntuación z, más intensa es la clusterización de valores altos. 
Una vez identificados los barrios con mayor concentración de VSMS se ha procedido a validar o rechazar la segunda hipótesis de la investigación (identificar si esta concentración de VSMS tiene lugar en las zonas que concentran mayor vulnerabilidad de la ciudad). Para la caracterización socioeconómica de los barrios se han utilizado dos indicadores: la renta media de los hogares en 2012 (información facilitada por el estudio del proyecto europeo Urban Audit de 2013) y el nivel de estudios de la población residente (datos del censo de población y vivienda de 2011). Urban Audit ofrece los datos agregados por subdistritos ${ }^{9}$, por lo que se ha homogeneizado la base de datos a esta escala. Para ello se han agregado los datos de nivel de estudios por secciones censales y se ha calculado el total de VSMS, VSMS por 1.000 habitantes y VSMS por 1.000 viviendas por subdistritos. A partir de estos datos se ha aplicado el coeficiente de correlación de Spearman, el cuál que permite ponderar el grado de asociación o interdependencia entre variables continuas independientes. El coeficiente se define mediante la fórmula:

$$
r_{s}=1-\frac{6 \Sigma d^{2}}{n\left(n^{2}-1\right)}
$$

Dónde $r_{s}$ es el coeficiente de correlación por rangos de Spearman, $d^{2}$ es la diferencia entre los rangos de las dos variables, elevadas al cuadrado, $n$ es el número de valores, y $\Sigma$ el sumatorio. Los resultados del estadístico de Spearman arrojan valores comprendidos entre +1 y -1 . Valores próximos al cero significan la ausencia de correlación estadística entre las variables estudiadas. Cuanto más próximo al 1 sea el resultado más fuerte será la correlación directa o positiva (el incremento de una implica el de la otra), mientras cuanto más próximo a -1 sea el resultado más intensa será la correlación inversa o negativa (el incremento de una implica el descenso de la otra o la inversa).

\section{Geografía de la desposesión de vivienda en España}

La distribución territorial de las VSMS puede definirse a partir de dos tendencias. En primer lugar, se constata que las grandes ciudades tienden a concentrar los principales volúmenes. Distribución lógica, aunque existen notables diferencias entre ciudades de tamaño similar. En segundo lugar, el litoral mediterráneo acumula una parte significativa de este stock (ver Figura No 1). Así pues, de las 33.205 VSMS identificadas a través de nuestra metodología, Cataluña (8.186 unidades) y la Comunidad Valenciana (9.255) acumulan el 52,5\% del total. Cifra que llega al 66,2\% si se incluye Andalucía (4.525 viviendas).

El mapa de la Figura № 1 permite identificar los territorios más afectados por la burbuja inmobiliaria y la sobreexposición al riesgo hipotecario. Por ello, el conjunto del litoral mediterráneo y la gran área metropolitana de Madrid son las regiones con mayor afectación. Como apuntan Burriel (2014) y Méndez y Plaza (2016), aquellos territorios que experimentar una mayor expansión del crédito asociado al ciclo inmobiliario alcista han sido los que, posteriormente, han concentrado un crecimiento acelerado de las ejecuciones hipotecarias. En este contexto, las zonas turísticas del litoral han jugado un papel central. Son zonas que han sufrido una presión urbanizadora y una burbuja de precios también especialmente altas. Si se expresan los datos en valores relativos,

En el caso de España las áreas submunicipales o subdistritos utilizados por Urban Audit se construyen mediante la agregación de secciones censales y con la condición de que cada uno sume entre 5.000 y 40.000 habitantes. 
Figura $\mathrm{N}^{\circ} 1$

Viviendas propiedad de la SAREB

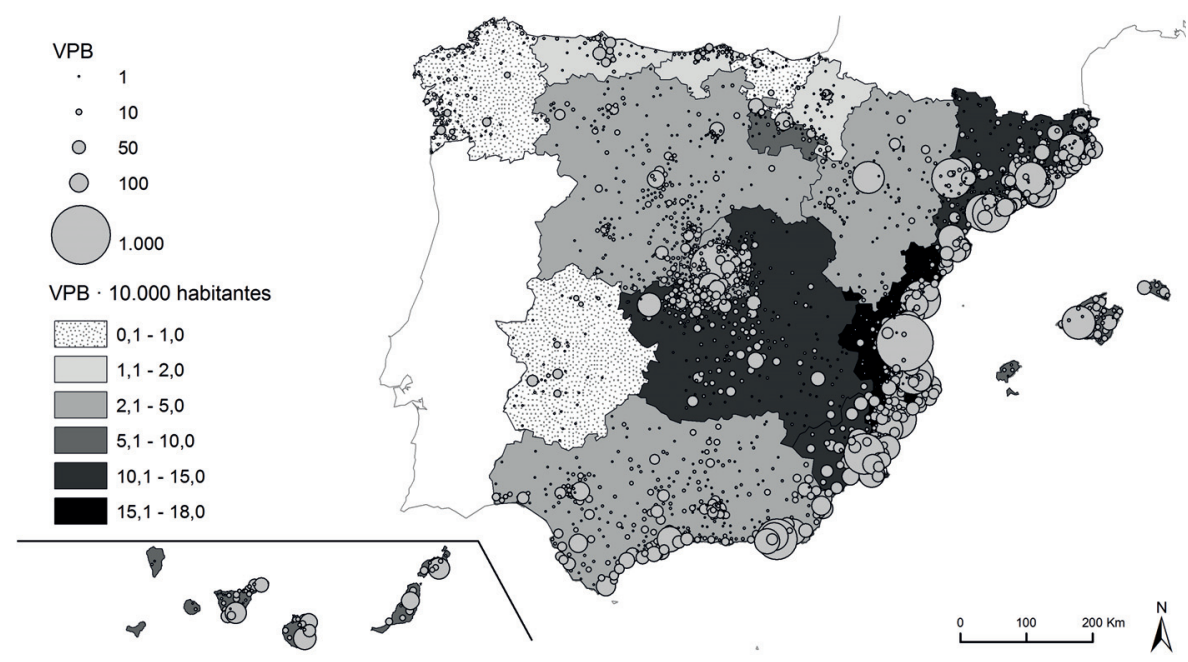

Fuente: Elaboración propia a partir de datos de los portales inmobiliarios de los bancos y del Censo de Población y Vivienda de 2011 (Instituto Nacional de Estadística).

utilizando el indicador VSMS por 10.000 habitantes, la Comunidad Valenciana presenta una cifra especialmente más elevada que el resto de comunidades (18,5 viviendas por 10.000 habitantes), seguida de la región de Murcia (13,7 viviendas por 10.000 habitantes), Cataluña y Castilla la Mancha (11 y 10,6 viviendas por 10.000 habitantes, respectivamente). Así, además del litoral mediterráneo, el sur metropolitano de Madrid (área de Castilla - La Mancha fronteriza con la Comunidad de Madrid) es otro espacio de alta concentración de VSMS. Por el contrario, el País Vasco, Cantabria, Navarra y Extremadura son las regiones con menor concentración de VSMS (ver Cuadro No 2 para más detalle). En términos de Vives-Miró et al. (2015), estos espacios turísticos y zonas de expansión metropolitana han resultado un escenario hegemónico para la apropiación de rentas urbanas a través de la financiarización del espacio construido. Esta financiarización y las expectativas de incremento de renta potencial por el "efecto riqueza" derivado del aumento de valor de las propiedades inmobiliarias han conducido a un proceso de endeudamiento masivo que ha conducido al presente proceso de desposesión, también masiva, de vivienda; conformando así un ciclo completo de acumulación por desposesión (Harvey, 2003). Por ello, cuanto más exposición a la burbuja inmobiliaria durante el ciclo alcista, más presión de los desahucios durante la crisis.

Cuadro No 2

Viviendas propiedad de la SAREB por Comunidades Autónomas

\begin{tabular}{|l|r|r|r|}
\hline & \multicolumn{1}{|c|}{ VSMS } & Población 2011 & \multicolumn{2}{|c|}{$\begin{array}{c}\text { VSMS por 10.000 } \\
\text { habitantes }\end{array}$} \\
\hline Comunidad Valenciana & 9.255 & 4.990 .345 & 18,5 \\
\hline Región de Murcia & 1.998 & 1.458 .250 & 13,7 \\
\hline Cataluña & 8.186 & 7.472 .935 & 11,0 \\
\hline
\end{tabular}


Continuación Cuadro No 2

\begin{tabular}{|l|r|r|r|}
\hline & VSMS & Población 2011 & $\begin{array}{c}\text { VSMS por 10.000 } \\
\text { habitantes }\end{array}$ \\
\hline Castilla - La Mancha & 2.219 & 2.092 .395 & 10,6 \\
\hline Illes Balears & 907 & 1.096 .905 & 8,3 \\
\hline La Rioja & 218 & 319.460 & 6,8 \\
\hline Canarias & 1.165 & 2.078 .280 & 5,6 \\
\hline Andalucía & 4.525 & 8.343 .655 & 5,4 \\
\hline Aragón & 642 & 1.331 .190 & 4,8 \\
\hline Castilla y León & 1.087 & 2.515 .755 & 4,3 \\
\hline Comunidad de Madrid & 2.016 & 6.387 .250 & 3,2 \\
\hline Navarra & 136 & 635.175 & 2,1 \\
\hline Cantabria & 124 & 589.175 & 2,1 \\
\hline Asturias & 222 & 1.069 .275 & 1,4 \\
\hline Extremadura & 150 & 1.097 .695 & 1,0 \\
\hline Galicia & 278 & 2.759 .890 & 0,4 \\
\hline País Vasco & 77 & 2.173 .265 & 7,2 \\
\hline Total & 33.205 & 46.410 .895 & \\
\hline
\end{tabular}

Nota: Sin datos disponibles para las ciudades autónomas de Ceuta y Melilla.

Fuente: Elaboración propia a partir de datos de los portales inmobiliarios de los bancos y del Censo de Población y Vivienda de 2011 (Instituto Nacional de Estadística).

\section{Desahucios y profundización de la desigualdad urbana}

Las tres ciudades seleccionadas como caso de estudio han permitido profundizar el análisis a escala urbana e identificar pautas de distribución espacial de las VSMS. Los resultados del estadístico de Getis-Ord $\mathrm{Gi}^{*}$ muestran que efectivamente se produce una concentración de VSMS en algunos barrios de estas ciudades. Ello permite validar la primera hipótesis: los desahucios por ejecución hipotecaria no presentan una distribución homogénea y/o aleatoria, sino que tienden a concentrarse en determinados barrios.

La Figura No 2 muestra los resultados para la ciudad de Alicante. Ésta demuestra que existen zonas de clara concentración de VSMS, formando los clústeres o puntos calientes de alta concentración. Las manzanas identificadas con un valor de $z$ (desviación típica) entre -1,65 y 1,65 son aquellas que no presentan una concentración estadísticamente significativa. Las manzanas con un valor comprendido entre 1,65 y 1,95 son un clúster de alta concentración de VSMS identificado con un nivel de significación estadística del $90 \%(p<0,1)$. Las que alcanzan valores entre 1,95 y 2,58 lo son al $95 \%(p<0,05)$. Mientras que las que alcanzan un valor $z$ superior el 2,58 son clústeres evidentes, con una confianza estadística del $99 \%(p<0,01)$. Los barrios identificados como puntos calientes a través del estadístico espacial estadístico de Getis-Ord Gi* son: Carolinas Altas, Colonia Requena-Juan XIII, Las 1000 viviendas, Virgen del Carmen-400-Nou Alacant, Virgen del Remedio. 
Figura No 2

Clústeres de alta concentración de VSMS (Alicante)

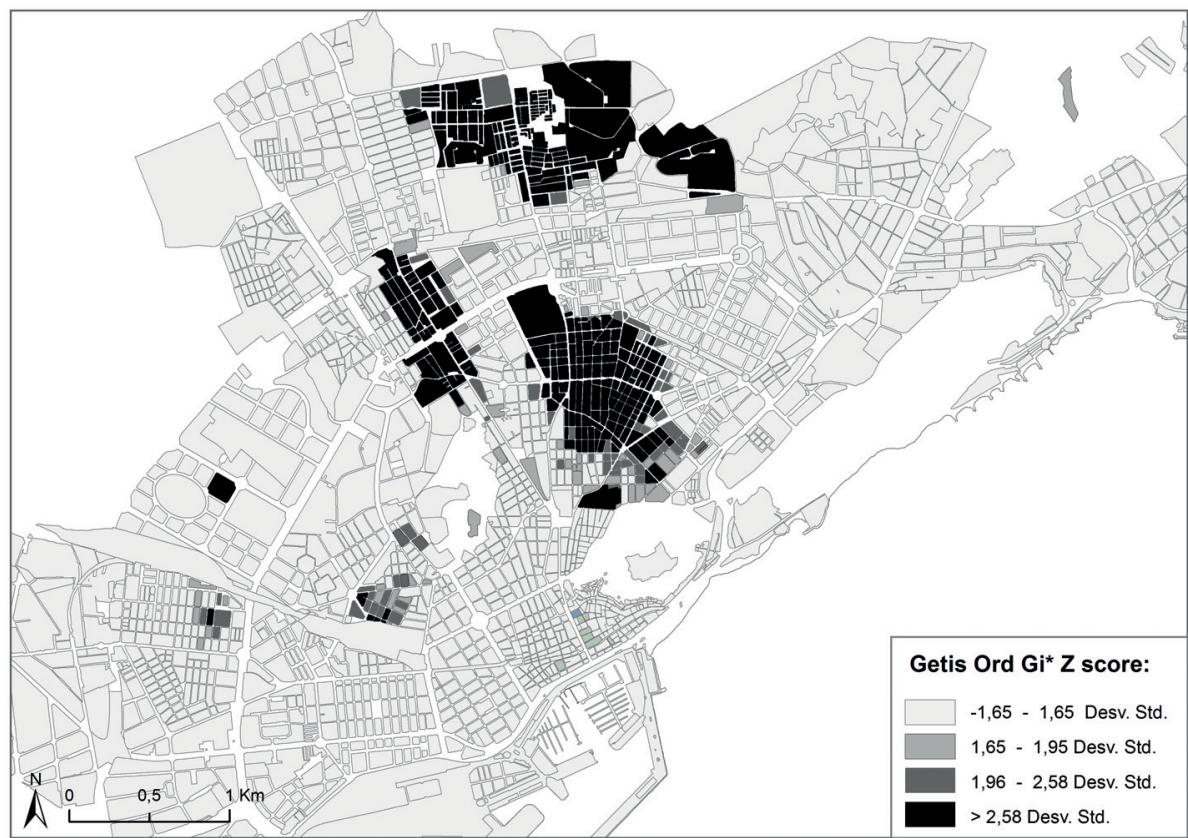

Fuente: Elaboración propia a partir de datos de los portales inmobiliarios de los bancos.

En el caso de Murcia (Figura $N^{\circ} 3$ ) los resultados obtenidos permiten identificar las zonas donde se concentran los desahucios por ejecución hipotecaria en los barrios céntricos de San Andrés, San Antolín y el Carmen, pero también en barrios periféricos del sur y del este de la ciudad. Se trata de los barrios de la Purísima de Barriomar (al suroeste del núcleo urbano), El Palmar y La Alberca (también en el suroeste, pero más periférico), Torreaguera y Los Ramos (al sureste) y Los Dolores, Puente Tocinos y Santa Cruz (este).

En Zaragoza (Figura $N^{\circ} 4$ ), también se ha podido constatar patrones claros de concentración. El barrio de San Pablo, en el casco histórico se define como un clúster de alta concentración de VSMS. También destacan La Almozara, Delícias, Parque Roma y Monsalud (al oeste) y La Paz, San José y Las Fuentes (al este y sureste). En el margen izquierdo del Ebro, los barrios de la Arrabal, el Picarral y la Jota son también definidos como importantes clústeres o puntos calientes.

Las evidencias aportadas en los mapas de las Figuras $\mathrm{N}^{\circ} 2, \mathrm{~N}^{\circ} 3$ y $\mathrm{N}^{\circ} 4$ permiten identificar, con precisión, clústeres de concentración de VSMS de alta significación estadística. De ello se deriva la pertinencia de validar la segunda hipótesis del trabajo. En este sentido, a modo de exploración inicial, cabe destacar que la mayor parte de estas zonas identificadas como clústeres se corresponden con aquellas que han sido definidas como barrios vulnerables por el Atlas de Barrios Vulnerables de España elaborado por el Ministerio de Fomento ${ }^{10}$; lo que aporta una pri-

10 http://atlasvulnerabilidadurbana.fomento.es/\#l=es;v=map2 
Figura $\mathrm{N}^{\circ} 3$

Clústeres de alta concentración de VSMS (Murcia)

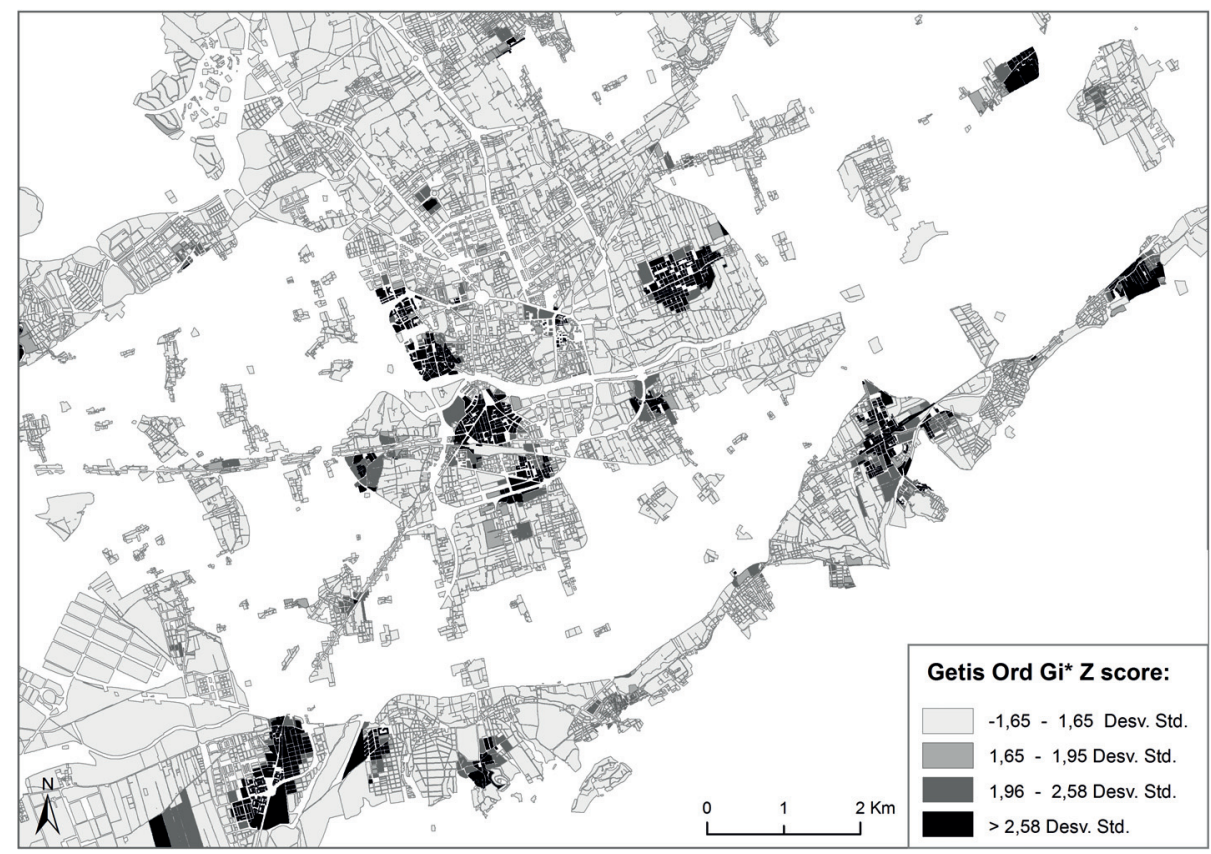

Fuente: Elaboración propia a partir de datos de los portales inmobiliarios de los bancos.

Figura No 4

Clústeres de alta concentración de VSMS (Zaragoza)

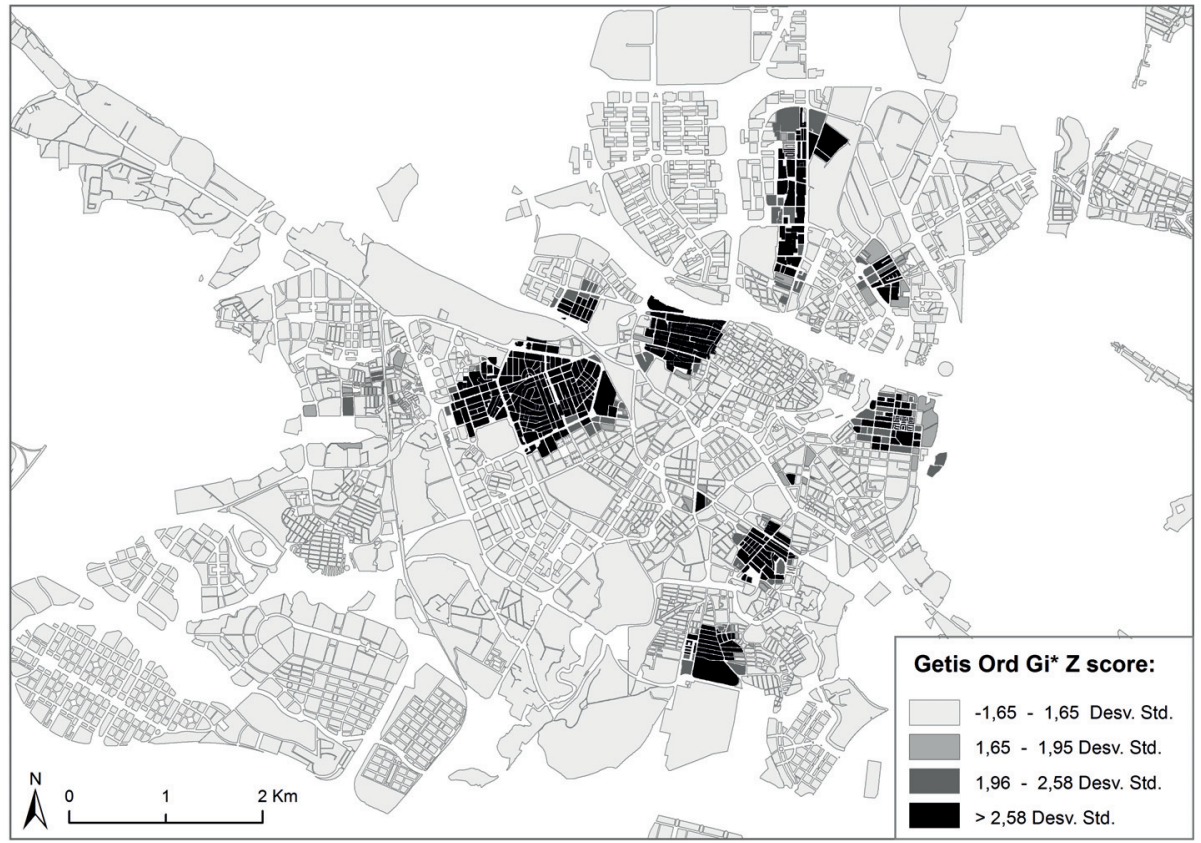

Fuente: Elaboración propia a partir de datos de los portales inmobiliarios de los bancos. 
mera evidencia del tipo de barrio donde han tendido a concentrarse los desahucios derivados de ejecución hipotecaria.

La Figura No 5 permite evidenciar la correlación entre los indicadores sociodemográficos utilizados (renta de los hogares y nivel de estudios) y la distribución espacial de las VSMS en las ciudades estudiadas. Como puede apreciarse, la pauta es clara en los tres casos: los subdistritos con menor renta (tramas blanca y gris claro de los mapas A) tienden a concentrar más VSMS; además, los subdistritos con mayor porcentaje de población analfabeta (tramas negra y gris oscuro de los mapas B) concentran también mayor número de VSMS. El tercer mapa en cada caso (mapa C), relaciona la distribución espacial de las VSMS con su peso relativo sobre el total de parque de vivienda en cada subdistrito. Este mapa demuestra que en las zonas donde se concentra un mayor número de VSMS, estas viviendas también tienen un mayor peso relativo sobre el total de vivienda del barrio. Es decir, que el número alto de VSMS no es debido, simplemente, al hecho que el barrio en cuestión posee un número elevado de viviendas. Lo que permite evidenciar que esta concentración de VSMS se produce tanto en términos absolutos, como relativos; reafirmando así su definición como clústeres o puntos calientes.

A pesar de que cada una de las ciudades analizadas presenta sus propias particularidades, la aplicación del coeficiente de correlación estadística de Spearman ha permitido dibujar un patrón común entre ellas. Por lo que podemos hacer una lectura transversal de los resultados obtenidos (ver Cuadro No 3 ) y afirmar que aquellos subdistritos con mayor concentración de VSMS presen-

Figura No 5

Distribución de VSMS y perfiles socioeconómicos de los subdistritos (Alicante, Murcia y Zaragoza)

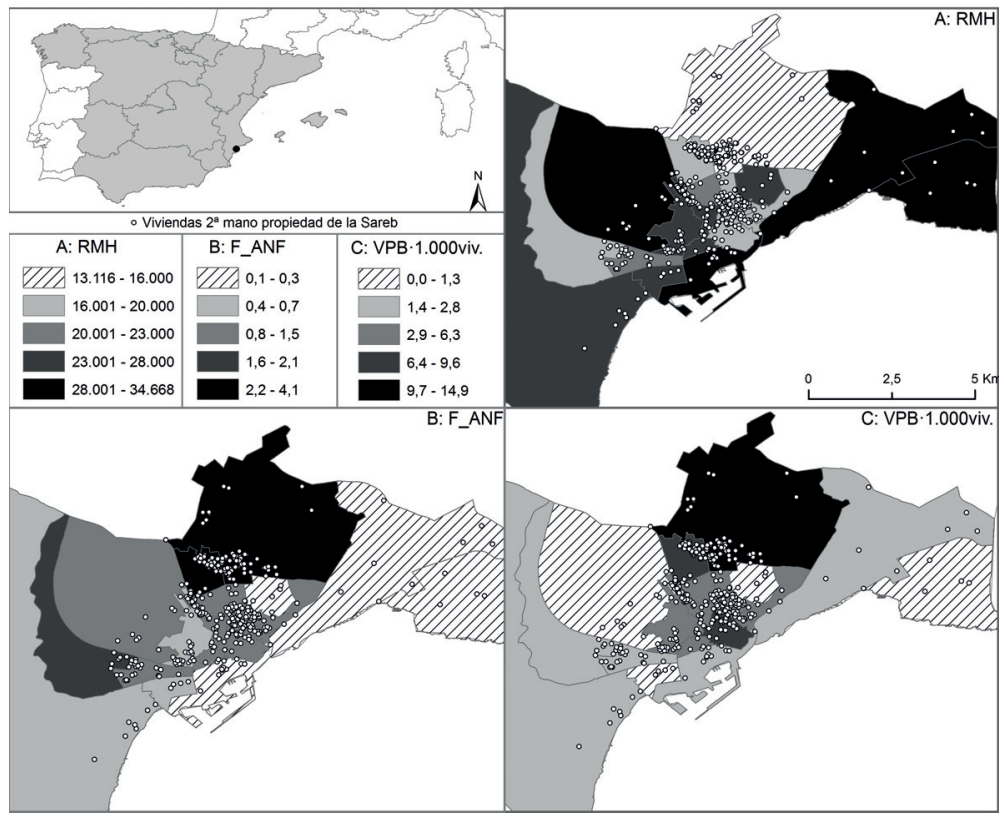



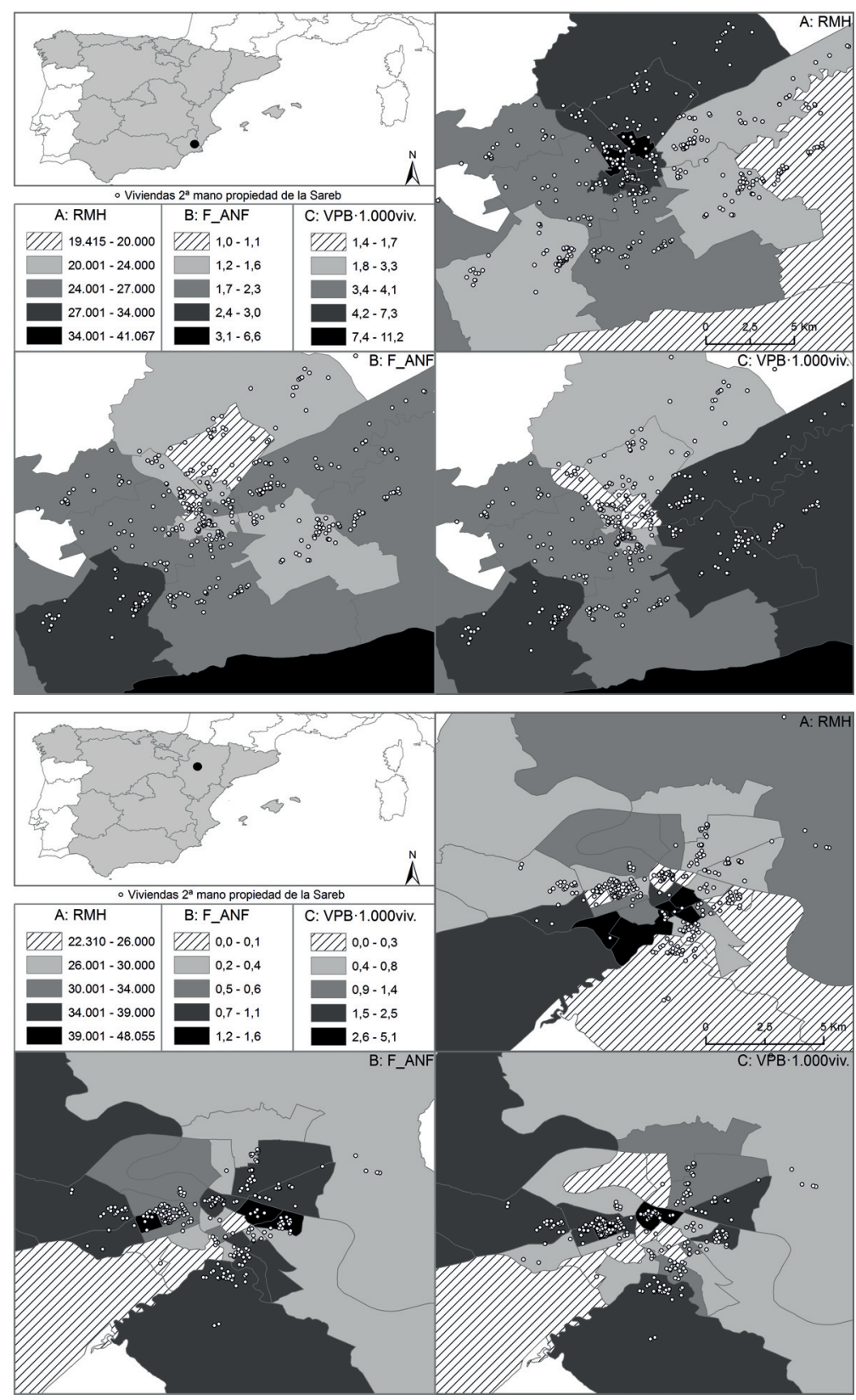

Nota 1: RMH - Renta media de los hogares; ANF - Porcentaje de población analfabeta; VSMS $\cdot 1.000$ viv. - Viviendas de segunda mano propiedad de la SAREB por cada 1.000 viviendas. Datos de las tres variables relativos a subdistritos.

Nota 2: Los intervalos, en cada ciudad, han sido definidos a partir del método de optimización de Jenks, que permite establecer intervalos según los puntos de ruptura naturales (natural breaks) de la distribución. Para la variable "porcentaje de analfabetos" y para el ratio de "VSMS por mil viviendas" se ha mantenido el intervalo que resulta de la aplicación de dicha técnica estadística. Para la RMH se ha redondeado al millar.

Fuente: Elaboración propia a partir de datos de los portales inmobiliarios de los bancos, del Censo de Población y Vivienda de 2011 (Instituto Nacional de Estadística) y de Urban Audit, 2013. 
tan una renda media por hogar más baja y un nivel formativo menor. Se trata, pues, de un patrón caracterizado por la concentración de los desahucios en aquellos barrios más vulnerables.

\section{Cuadro $\mathrm{N}^{\circ} 3$}

Correlación estadística de Spearman entre el volumen VSMS y variables socioeconómicas de los subdistritos

\begin{tabular}{|c|c|c|c|c|c|c|}
\hline & & $\sum_{\infty}^{\infty}$ & $\begin{array}{l}\bar{\delta} \\
\text { N } \\
\infty^{\prime} \\
O \\
\Sigma\end{array}$ & $\begin{array}{l}\overparen{\Psi} \\
\sum_{\alpha}^{I}\end{array}$ & 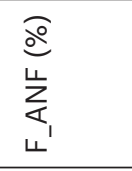 & $\begin{array}{l}\widehat{\curvearrowright} \\
z_{m} \\
\iota_{1}\end{array}$ \\
\hline \multirow{6}{*}{ 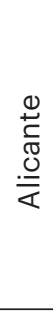 } & Max. & 81 & 37.355 & 34.668 & 4,1 & 57,9 \\
\hline & Min. & 4 & 9.645 & 13.116 & 0,1 & 3,7 \\
\hline & Promedio & 23,8 & 19.300 & 24.131 & 1,2 & 17,7 \\
\hline & Desv.Std. & 20,8 & 6.684 & 6.084 & 1,1 & 13,9 \\
\hline & \multirow{2}{*}{ r Spearman } & \multicolumn{2}{|c|}{ VSMS por 1.000 hab. } &,$- 865\left(^{* *}\right)$ &, $711(* *)$ &,$- 699(* *)$ \\
\hline & & \multicolumn{2}{|c|}{ VSMS por 1.000viv } &,$- 797\left(^{* *}\right)$ & $657(* *)$ &,$- 632(* *)$ \\
\hline \multirow{6}{*}{$\begin{array}{l}\frac{.0}{0} \\
\frac{\stackrel{3}{2}}{\Sigma}\end{array}$} & Max. & 55 & 39.355 & 41.067 & 6,6 & 34,2 \\
\hline & Min. & 9 & 11.015 & 19.415 & 1,0 & 6,7 \\
\hline & Promedio & 28,3 & 27.280 & 27.486 & 2,0 & 17,5 \\
\hline & Desv.Std. & 14,3 & 6.818 & 5.969 & 1,4 & 9,0 \\
\hline & \multirow{2}{*}{ r Spearman } & \multicolumn{2}{|c|}{ VSMS por 1.000hab. } &,$- 882\left(^{* *}\right)$ &, $503\left(^{*}\right)$ &,$- 924(* *)$ \\
\hline & & \multicolumn{2}{|c|}{ VSMS por 1.000viv } &,$- 897\left(^{* *}\right)$ &, $526\left(^{*}\right)$ &,$- 924(* *)$ \\
\hline \multirow{6}{*}{ 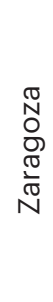 } & Max. & 41 & 36.420 & 48.055 & 1,6 & 44,8 \\
\hline & Min. & 0 & 8.625 & 22.311 & 0,0 & 7,3 \\
\hline & Promedio & 9,2 & 21.030 & 31.395 & 0,6 & 21,2 \\
\hline & Desv.Std. & 9,8 & 6.263 & 6.866 & 0,4 & 9,9 \\
\hline & r spearman & \multicolumn{2}{|c|}{ VSMS por 1.000hab. } &,$- 761\left(^{* *}\right)$ &, $536\left(^{* *}\right)$ &,$- 594(* *)$ \\
\hline & rspearman & \multicolumn{2}{|c|}{ VSMS por 1.000viv } &,$- 726\left(^{* *}\right)$ &, $533\left({ }^{* *}\right)$ &,$- 588(* *)$ \\
\hline
\end{tabular}

${ }^{*}$ Correlación significativa $p<0,01$. ${ }^{*}$ Correlación significativa $p<0,05$.

Nota: POB_2011 - Población del municipio, según Censo de Población y Vivienda de 2011; RMH - Renta media de los hogares; ANF - Porcentaje de población analfabeta; F_3N - Porcentaje de población con estudios superiores (universitarios); VSMS $\cdot 1.000$ hab. - Viviendas de segunda mano propiedad de la SAREB por cada 1.000 habitantes; VSMS $\cdot 1.000$ viv. - Viviendas de segunda mano propiedad de la SAREB por cada 1.000 viviendas. Datos de las todas las variabes relativos a subdistritos.

Fuente: Elaboración propia a partir de datos de los portales inmobiliarios de los bancos, del Censo de Población y Vivienda de 2011 (Instituto Nacional de Estadística) y de Urban Audit, 2013.

Los resultados del estadístico de Spearman entre la mayor o menor presencia de VSMS, la renta media de los hogares y el nivel de estudios por subdistritos arrojan unas fuertes correlaciones en las tres ciudades y para ambas variables. Además, los resultados son estadísticamente significativos en todos los casos. La correlación negativa en relación al nivel de renta es clara. A 
mayor nivel de renta media, menor VSMS por 1.000 habitantes o por 1.000 viviendas $y$, a menor nivel de renda, mayor volumen de VSMS. Los valores de correlación negativa oscilan entre -0,761 (Zaragoza) y -0,882 (Murcia) en relación a las VSMS por 1.000 habitantes y -0,726 (Zaragoza) y $-0,897$ (Murcia) en relación a las VSMS por 1.000 viviendas $(p<0,01)$. La misma lógica se dibuja en la relación con la distribución de VSMS y la población con estudios superiores. Alcanza una correlación inversa o negativa importante, aunque con un umbral mínimo inferior al anterior. Así, los valores de correlación oscilan entre -0,594 (Zaragoza) y -0,924 (Murcia) en relación a las VSMS por 1.000 habitantes y -0,588 (Zaragoza) y -0,924 (Murcia) en relación a las VSMS por 1.000 viviendas $(p<0,01)$. Finalmente, la presencia de población analfabeta y el número de VSMS presentan una correlación positiva (mayor presencia de esta población supone, también, mayor presencia de VSMS). En este caso, cabe destacar que la correlación estadística alcanzada, aunque también es importante, alcanza un nivel menor a las anteriores. En este caso, los valores de correlación oscilan entre 0,503 (Murcia, $\mathrm{p}<0,05$ ) y 0,711 (Alicante, $\mathrm{p}<0,01$ ) en relación a las VSMS por 1.000 habitantes y 0,526 (Murcia $p,<0,05$ ) y 0,657 (Alicante, $p<0,01$ ) en relación a las VSMS por 1.000 viviendas $(p<0,01)$. Esta menor correlación y nivel de confianza estadística puede argumentarse a partir de la heterogeneidad de componentes que explican la tasa de analfabetismo en España. Ésta, además de estar relacionada con el perfil socioeconómico de la población, deriva también de la mayor o menor presencia de población más envejecida; ya que en España el analfabetismo tiende a concentrarse en las generaciones más pretéritas.

Además de estas correlaciones, cabe apuntar que la ciudad de Alicante es la que presenta mayores contrastes, con unos valores extremos más alejados y una mayor desviación estándar y, por tanto, mayores desigualdades entre subdistritos (máximo de VSMS por subdistrito: 81; mínimo de VSMS por subdistrito: 4; desviación estándar: 20,8); mientras que Murcia es la que presenta unos valores globales y medios más elevados (total de VSMS: 454; promedio por subdistrito: 28,3 ), por tanto es la ciudad que ha experimentado un proceso más intenso de desposesión de vivienda.

\section{Conclusiones}

La SAREB acumula en su propiedad un total de 33.205 viviendas vacías que, previamente, habían sido adquiridas por bancos españoles a través de ejecuciones hipotecarias que habían derivado en el desahucio de las familias que residían en ellas. Estas viviendas han llegado a manos de la SAREB en el marco del proceso de reestructuración y saneamiento del sector financiero español, completando así un proceso de acumulación de capital por desposesión masiva de viviendas auspiciado con políticas y recursos públicos.

El análisis de la distribución territorial de estas viviendas ha permitido identificar el litoral mediterráneo y el gran ámbito metropolitano de Madrid (especialmente la vertiente sur) como los territorios más afectados por este proceso de desposesión de vivienda. Éstos son los territorios más afectados por la hipertrofia inmobiliaria, la burbuja de precios y la sobreexposición al riesgo crediticio. Cuanta más exposición a los efectos del boom inmobiliario y burbuja de precios, mayor intensidad de desahucios por ejecución hipotecaria. Dinámica que evidencia como ambas cuestiones forman parte indiscernible del mismo proceso de generación y apropiación de rentas urbanas.

El análisis a escala urbana, basado en los casos de estudio de Alicante, Murcia y Zaragoza, ha evidenciado como las VSMS tienden a concentrarse en determinados barrios, los cuáles se han 
identificado como clústeres a través el análisis espacial desarrollado a escala de manzanas urbanas. Además, se ha demostrado una fuerte correlación negativa entre el nivel de renta y estudios y la concentración de VSMS por subdistritos. Es decir, a mayor renta y nivel de estudios, menor volumen de VSMS. Por el contrario, se ha evidenciado como los barrios y áreas urbanas que concentran población con menores recursos económicos son, precisamente, los que, con mayor intensidad resultan víctimas de esta desposesión masiva de vivienda.

El trabajo demuestra la relevancia de incorporar la dimensión espacial en el estudio de la crisis hipotecaria española. Los datos permiten demostrar empíricamente como los desahucios causados por ejecución hipotecaria no han tenido una distribución homogénea o aleatoria en las ciudades, sino que se ha concentrado en los colectivos y barrios más precarios, profundizando su situación de vulnerabilidad. De ello se desprende que la crisis hipotecaria está contribuyendo, significativamente, a profundizar la desigualdad en las ciudades españolas.

A su vez, la acumulación de este gran volumen de viviendas, y otros activos inmobiliarios, por parte de la SAREB ha conllevado el surgimiento de un nuevo gran propietario con una posición estratégica en el mercado inmobiliario español. Ello le convierte en un actor clave en el escenario post-crisis inmobiliaria. Por lo que investigaciones futuras deben abordar el estudio de la comercialización de su cartera inmobiliaria y si de ello deriva, como el escenario actual apunta, el aterrizaje de grandes inversores en búsqueda de una segunda ola de revalorización.

\section{Referencias bibliográficas}

AALBERS, M. The financialization of home and the mortgage market crisis. Competition \& Change, 2008, Vol. 12, No 2, p. 148-166.

ÁLVAREZ, E.; ZAPATA, M.J. \& ZAPATA, P. Stop the evictions! The diffusion of networked social movements and the emergence of a hybrid space: The case of the Spanish Mortgage Victims Group. Habitat International, 2015, Vol. 46, p. 252-259.

BARBERO, I. When rights need to be (re)claimed: Austerity measures, neoliberal housing policies and anti-eviction activism in Spain. Critical Social Policy, 2015, Vol. 35, № 2, p. 270-280.

BURRIEL, E. El estallido de la burbuja inmobiliaria y sus efectos en el territorio. En: ALBERTOS, J.M. y SÁNCHEZ, J.L. (Coordinadores). Geografía de la crisis económica en España. Valencia: Publicaciones Universidad de Valencia, 2014, p. 101-140.

CANO, G.; ETXEZARRETA, A.; DOL, K. \& HOEKSTRA, J. From Housing Bubble to Repossessions: Spain Compared to Other West European Countries. Housing Studies, 2013, Vol. 28, No 8, p. 11971217.

CHRISTOPHERS, B. The limits to financialization. Dialogues in Human Geography, 2015, Vol. 5, № $2, \mathrm{p}-183-200$.

COLAU, A. y ALEMANY, A. Vidas hipotecadas. De la burbuja inmobiliaria al derecho a la vivienda. Barcelona: Angle Editorial, Cuadrilátero Libros, 2012. 
COQ-HUELVA, D. Urbanisation and Financialisation in the Context of a Rescaling State: The Case of Spain. Antipode, 2013, Vol. 45, No 5, p. 1213-1231.

FERNÁNDEZ DURÁN, R. El tsunami urbanizador español y global: sobre sus causas y repercusiones devastadoras, y la necesidad de prepararse para el previsible estallido de la burbuja inmobiliaria. Barcelona: Virus editorial, 2006.

FERNÁNDEZ, A. y CRUZ, E. Territorio y actividad constructora: del "tsunami" a la crisis. Factores explicativos y propuesta de indicadores a escala municipal en Andalucía. Boletín de la Asociación de Geógrafos Españoles, 2011, Vol. 56, p.79-110.

GAJA, F. Tras el tsunami inmobiliario. Salir del atolladero. En: LÓPEZ, I. y RODRÍGUEZ, E. (coordinadores). Paisajes devastados. Después del ciclo inmobiliario: Impactos regionales y urbanos de la crisis. Madrid: Traficantes de sueños, 2013, p. 313-354.

GILI, M.; ROCA, M.; BASU, S.; MCKEE, M. \& STUCKLER, D. The mental health risks of economic crisis in Spain: evidence from primary care centres, 2006 and 2010. European Journal of Public Health, 2012, Vol. 23, No 1, p. 103-108.

GOTHAM, K.F. Creating liquidity out of spatial fixity: The secondary circuit of capital and the subprime mortgage crisis. International Journal of Urban and Regional Research, 2009, Vol. 32, No 2, p. 355-371.

GUTIÉRREZ, A. y DELCLÒS, X. ¿Hipertrofia inmobiliaria? Análisis de las pautas territoriales del boom e implicaciones del estallido de la burbuja en Cataluña. Cuadernos Geográficos, 2015, Vol. 54, No 1, p.283-306.

GUTIÉRREZ, A. \& DELCLÒS, X. The uneven distribution of evictions as new evidence of urban inequality: A spatial analysis approach in two Catalan cities. Cities, 2016, Vol. 58, p. 101-108.

GUTIÉRREZ, A. y DELCLÒS, X. Geografía de la crisis inmobiliaria en Cataluña: Una lectura a partir de los desahucios por ejecución hipotecaria. Scripta Nova. Revista Electrónica de Geografía y Ciencias Sociales, 2017, Vol. 21, № 557.

GUTIÉRREZ, A. \& DOMĖNECH, A. The Spanish mortgage crisis: Evidence of the concentration of foreclosures in the most deprived neighbourhoods. Die Erde - Journal of the Geographical Society of Berlin, 2017, Vol. 148, No 1, p. 39-57.

HARVEY, D. The New Imperialism. Oxford: Oxford University Press, 2003.

HOEKSTRA, J. \& VAKILI-ZAD, C. High vacancy rates and rising house prices: The Spanish paradox. Tijdschrift voor economische en sociale geografie, 2011, Vol. 102, No 1, p. 55-71.

LAPAVITSAS, C. Financialised capitalism: Crisis and financial expropriation. Historical Materialism, 2009, Vol. 17, No 2, p. 114-148. 
LAPAVITSAS, C. Theorizing financialization. Work, employment and society, 2011, Vol. 25, No 4, p. 611-626.

LÓPEZ, I. y RODRÍGUEZ, E. Fin de ciclo. Financiarización, territorio y sociedad de propietarios en la onda larga del capitalismo hispano (1959-2010). Madrid: Traficantes de sueños, 2010.

LÓPEZ, I. \& RODRÍGUEZ, E. The Spanish Model. New Left Review, 2011, No 69, p. 5-28.

MÉNDEZ, R y PLAZA, J. Crisis inmobiliaria y desahucios hipotecarios en España: una perspectiva geográfica. Boletín de la Asociación de Geógrafos Españoles, 2016, № 71, p. 99-127.

NAREDO, J.M. El modelo inmobiliario español y sus consecuencias. Boletín $C F+S, 2010, N^{\circ} 44$, p. 13-27.

OBESO, I. Análisis geográfico de los desahucios en España. Ería, 2014, No 95, p. 327-342.

ROLNIK, R. Late neoliberalism: the financialization of homeownership and housing rights. International Journal of Urban and Regional Research, 2013, Vol. 37, No 3, p. 1058-1066.

ROMANOS, E. Evictions, Petitions and Escraches: Contentious Housing in Austerity Spain. Social Movement Studies, 2014, Vol. 13, № 2, p. 296-302.

ROMERO, J.; JIMÉNEZ, F. \& VILLORIA, M. (Un)sustainable territories: causes of the speculative bubble in Spain (1996-2010) and its territorial, environmental, and sociopolitical consequences. Environment and Planning C: Government and Policy, 2012, Vol. 30, p. 467-486.

VALIÑO, V. (Coordinador). Emergencia habitacional en el Estado español: La crisis de las ejecuciones hipotecarias y los desalojos desde una perspectiva de los derechos humanos. Barcelona: Observatorio DESC y Plataforma de Afectados por la Hipoteca, 2013.

VALIÑO, V. (Coordinador). Emergència habitacional a Catalunya. Impacte de la crisi hipotecària en el dret a la salut i els drets dels infants. Barcelona: Observatorio DESC y Plataforma de Afectados por la Hipoteca, 2015.

VINUESA, J. El festín de la vivienda. Auge y caída del negocio inmobiliario en España. Madrid: Díaz \& Pons, 2013.

VIVES-MIRÓ, S.; GONZÁLEZ-PÉREZ, J.M. \& RULLAN, O. Home dispossession: the uneven geography of evictions in Palma (Majorca). Die Erde. Journal of the Geographical Society of Berlin, 2015, Vol. 146, No 2-3, 113-126.

VIVES-MIRÓ, S. y RULLAN, O. La apropiación de las rentas del suelo en la ciudad neoliberal española. Boletín de la Asociación de Geógrafos Españoles, 2014, № 65, p. 387-408. 